Randomised controlled trial

\section{Calcaneal fractures have universally poor outcomes regardless of management}

\subsection{6/ebmed-2014-110088}

\section{Tun Hing Lui, Samuel Ka Kin Ling}

Department of Orthopaedics and Traumatology, North District Hospital, 999077, Hong Kong

Correspondence to: Dr Tun Hing Lui, Department of Orthopaedics and Traumatology, North District Hospital, 9 Po Kin Road, Sheung Shui, Hong Kong, Hong Kong; luithderek@yahoo.co.uk

Commentary on: Griffin D, Parsons N, Shaw E, et al. Operative versus non-operative treatment for closed, displaced, intra-articular fractures of the calcaneus: randomised controlled trial. BMJ 2014;349:g4483.

\section{Context}

Calcaneal fractures have a significant socioeconomic impact, because they usually occur in working-age individuals after a relatively highenergy trauma. As stated in this study, these fractures are notoriously difficult to manage and the orthopaedic community continues to debate between conservative and operative management. ${ }^{1}$ Several systematic reviews have been performed, but none have presented overwhelming advice on which is the superior option. ${ }^{2}$

\section{Method}

This is a multicentre, allocation concealed, randomised controlled trial comparing conservative and operative management of closed displaced intra-articular calcaneal fractures. In the operative arm, the standard extensile lateral approach with open reduction and internal fixation was chosen. In the conservative arm, the patients were given a splint without any attempt at reduction; they progressed from non-weight-bearing walking, to partial weight-bearing walking and then full weight-bearing walking at 6-week intervals. All participants underwent the same rehabilitation regime and outcomes were measured by: functional scoring, radiographs and the need for further intervention.

The catchment consisted of 2006 patients over 2 years, which was nearly $50 \%$ of all calcaneal fractures in the UK. An interesting note was that eligible patients whom declined entry into the study, were subsequently managed half by surgery and half by conservative measures.

\section{Findings}

This study found no significant difference between the two treatment arms, with both groups having uniformly poor function, refuting previous studies which claimed better outcomes with operations in certain fracture patterns. The major morbidity in the operative patients was soft tissue and wound complications, while the conservative group had significant subtalar joint arthritis.

\section{Commentary}

The 156 recruited cases were a diverse patient pool with multiple uncontrolled variables, such as injury mechanism, severity, fracture patterns and surgical timing. The authors performed a pilot study with a power analysis to show that their overall data should be statistically significant. However, because other studies have claimed benefits in operative management for specific subtypes of calcaneal fractures, a larger sample size would have allowed more accuracy in further prespecified subgroup analysis. $^{3}$

Surgeons operating on an average of one case per year performed most of the procedures; this leads to the postulation that perhaps surgeries performed in higher volume centres may have better functional outcomes. The timing of the surgery was $1-3$ weeks postinjury, which is generally acceptable. However, some advocate that urgent surgery prior to the onset of swelling may be more beneficial for the soft tissue. This drains resources, but since soft tissue problems are the most important surgical complication, the added emergency workload may be justifiable.

The relatively short follow-up of 2 years leaves some hesitation about long-term outcomes. Since secondary post-traumatic subtalar arthritis is expected to deteriorate with time, it is entirely likely that more participants in the conservative group will have significant progression of arthritis. Follow-up studies at 5 or 10 years will be interesting-as long as the number of participants is kept above 126 as calculated in the power analysis.

Despite some limitations, this is a well-performed study, which has the frustrating conclusion that efforts to develop surgery for calcaneal fractures may have been futile. The silver lining is that we have braved new ground with improved techniques on dealing with complications, such as preliminary successes with arthroscopic assisted subtalar joint arthrodesis and endoscopic calcaneoplasties. ${ }^{4}$ The result also encourages us to be more innovative in pursuit of fixation methods with less soft tissue trauma, such as percutaneous fixation. We should continue to strive and help our patients regain meaningful function so that they will enjoy uniformly good outcomes after calcaneal fractures, not uniformly poor ones.

Contributors SKKL has reviewed the article and drafted the commentary. THL has supervised and revised the work.

Competing interests None.

Provenance and peer review Commissioned; internally peer reviewed.

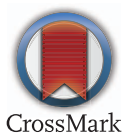

\section{References}

1. Bruce J, Sutherland A. Surgical versus conservative interventions for displaced intra-articular calcaneal fractures. Cochrane Database Syst Rev 2013; 1:CD008628.

2. Ibrahim T, Rowsell M, Rennie W, et al. Displaced intra-articular calcaneal fractures: 15-year follow-up of a randomised controlled trial of conservative versus operative treatment. Injury 2007;38:848-55.

3. Basile A. Operative versus nonoperative treatment of displaced intra-articular calcaneal fractures in elderly patients. J Foot Ankle Surg 2010; 49:25-32.

4. Lui TH. New technique of arthroscopic triple arthrodesis. Arthroscopy 2006;22:464. e1-5. 